\title{
A efetividade do tratamento da dor no pós operatório de cirurgias ortopédicas
}

\author{
The treatment effectiveness dolor en el postoperatorio de cirugías ortopédicas
}

La eficacia del tratamiento pain in the postoperative period of orthopedic surgeries

Carina Galvan1, Rosaura Soares Paczek ${ }^{1 *}$, Bruna Noschang de Brum², Débora Machado Nascimento do Espírito Santo ${ }^{1}$, Ana Karina Silva da Rocha Tanaka², Daniela Trintinaia Brito².

\section{RESUMO}

Objetivo: Avaliar e refletir sobre a dor no pós operatório de cirurgias ortopédicas através de uma revisão narrativa da literatura. Revisão bibliográfica: A dor é definida como experiência sensorial e emocional desagradável associada à lesão tecidual, podendo ser aguda ou crônica. Pode estar relacionada à frustração, raiva e depressão, crença, expectativa e percepção do paciente na sua condição. A dor no pré-operatório, duração da doença e ansiedade pós-cirúrgica são considerados preditivos para o desenvolvimento de dor crônica no pós-operatório de cirurgia ortopédica. O controle da dor no pós-operatório envolve prescrição de analgésicos em horários fixos, uso de métodos sofisticados como cateteres peridurais; evitando o sofrimento, proporcionando maior satisfação com o atendimento e reduzindo os custos relacionados a possíveis complicações que resultam num período prolongado de internação. A analgesia efetiva resulta numa recuperação mais rápida e maior colaboração com o tratamento, com resultados positivos no pós-operatório. Considerações finais: $O$ enfermeiro possibilita que o paciente exponha suas preocupações e sentimentos do processo cirúrgico como um todo. O ortopedista e o enfermeiro possuem papel fundamental para avaliar, controlar e aliviar a dor no tratamento eficaz do trauma, pois acelera o processo de reabilitação e contribui na manutenção das funções fisiológicas do paciente.

Palavras-chave: Manejo da dor, Cuidados pós-operatórios, Ortopedia, Analgesia.

\section{ABSTRACT}

Objective: To evaluate and reflect about pain in the postoperative period of orthopedic surgeries through a narrative review of the literature. Literature review: The pain is defined as the unpleasant sensory and emotional experience associated with tissue injuries, which can be acute or chronic. It may be influence by frustration, anger and depression, beliefs, expectation and perception of the patient of his condition. Preoperative pain, disease and post-surgical anxiety are considered predictive for the development of chronic pain in the postoperative period of orthopedic surgery. Postoperative pain control involves prescribing analgesics at fixed times, use of sophisticated methods such as epidural catheters; avoid suffering, decrease satisfaction with care and limit costs related to possible complications resulting from a prolonged period of hospitalization. Effective analgesia results in faster recovery and greater collaboration with treatment, implying in positive postoperative results. Final considerations: The nurse allows the patient expose his concerns and feelings about the entire surgical process. The orthopedist surgeon and the nurse have a fundamental role to assess, control and relieve pain in the effective treatment of trauma, as it speeds up the rehabilitation process and helps to maintain the patient's physiological functions.

Keywords: Pain management, Postoperative care, Orthopedics, Analgesia.

\section{RESUMEN}

Objetivo: Evaluar y reflexionar sobre el dolor en el postoperatorio de cirugías ortopédicas mediante una revisión narrativa de la literatura. Revisión bibliográfica: El dolor se define como una experiencia sensorial y emocional desagradable asociada con daño tisular, que puede ser aguda o crónica. Puede estar relacionado con la frustración, la ira y la depresión, las creencias, las expectativas y la percepción del paciente en su condición. El dolor preoperatorio, la duración de la enfermedad y la ansiedad postoperatoria se consideran predictivos para el desarrollo de dolor crónico en el período postoperatorio de la cirugía ortopédica. El control

1 Hospital de Clínicas de Porto Alegre, Porto Alegre - RS. *E-mail: rspaczek@gmail.com
2 Universidade Federal do Rio Grande do Sul (UFRGS), Porto Alegre - RS.

SUBMETIDO EM: 8/2020

ACEITO EM: 9/2020

PUBLICADO EM: 10/2020

REAS/EJCH | Vol.12(10) | e4875 | DOI: https://doi.org/10.25248/reas.e4875.2020 Página 1 de 7 
del dolor postoperatorio implica la prescripción de analgésicos a horas fijas, utilizando métodos sofisticados como los catéteres epidurales; evitando el sufrimiento, proporcionando una mayor satisfacción con la atención y reduciendo los costos relacionados con posibles complicaciones que resultan en un período prolongado de hospitalización. La analgesia efectiva resulta en una recuperación más rápida y una mayor colaboración con el tratamiento, con resultados postoperatorios positivos. Consideraciones finales: La enfermera le permite al paciente exponer sus preocupaciones y sentimientos sobre el proceso quirúrgico en su conjunto. El ortopedista y la enfermera tienen un papel fundamental en la evaluación, el control y el alivio del dolor en el tratamiento eficaz del trauma, ya que acelera el proceso de rehabilitación y contribuye al mantenimiento de las funciones fisiológicas del paciente.

Palabras clave: Manejo del dolor, Cuidados posoperatorios, Ortopedia, Analgesia.

\section{INTRODUÇÃO}

A hospitalização interfere na rotina do paciente, levando-o a um lugar desconhecido com rotinas e pessoas desconhecidas, levando ao aumento do estresse da ansiedade afetando o seu emocional (BARROS EA, 2018). A dor pode ser experienciada de forma sensitiva e emocional em decorrência de lesões nos tecidos.

Ao sofrer algum tipo de lesão, as células teciduais acometidas liberam prostaglandinas que causam hipersensibilidade, transformando o mínimo estímulo em dor (BARBOSA MH, et al., 2014). É um sintoma comum como parte do mecanismo de defesa do organismo, estando relacionada com a cirurgia propriamente dita, ao grau de traumatismo, ao tipo de incisão e ao posicionamento cirúrgico prolongado. Está presente em qualquer articulação com restrição de movimentos (SOARES AB, et al., 2013).

O procedimento cirúrgico ocasiona um trauma acarretando alterações fisiológicas e emocionais, que necessitam controle, senão podem ocorrer complicações, aumentando o tempo de permanência no ambiente hospitalar, e apesar dos avanços na medicina a dor ainda é um sintoma muito mencionado no pós-operatório, merecendo destaque no cuidado, pois é de fácil entendimento, mas complexa para ser avaliada devido a sua subjetividade (SALGADO PO, et al., 2017).

A cirurgia ortopédica e traumatológica está associada à dor pós-operatória severa, decorrente da intensa estimulação nociceptiva do tecido musculoesquelético e da analgesia pós-operatória inadequada. Essa situação acaba causando um desconforto significativo e efeitos físicos e psicológicos negativos no paciente, além de grande impacto socioeconômico por aumentar a morbidade e o tempo de internação hospitalar (GEMMA R, et al., 2014).

$\mathrm{Na}$ Sala de Recuperação Pós-Anestésica (SRPA) a equipe de enfermagem presta cuidados intensivos e semi-intensivos que auxiliam na recuperação da consciência, da estabilidade dos sinais vitais, da motilidade e da homeostase; é o período do pós-operatório imediato que inicia desde a saída da sala cirúrgica até a alta da sala de recuperação (BARBOSA MH, et al.,2014).

$A$ anestesia é essencial na escolha da analgesia no pós-operatório. Depende de fatores relacionados às características do paciente, à experiência do anestesiologista e às exigências da cirurgia e da reabilitação. $O$ paciente tem expectativas altas após a cirurgia e, às vezes, atividades rotineiras como vestir-se, caminhar, subir ou descer um lance de escada acabam sendo um desafio ainda maior por causa da dor no pósoperatório (PEREIRA DL, et al., 2016).

O objetivo deste estudo é realizar uma revisão narrativa da literatura sobre a dor no pós operatório de cirurgias ortopédicas, refletindo sobre as intervenções de enfermagem que podem ser realizadas para melhora da dor, auxiliando na recuperação do paciente para o retorno às suas atividades de vida diária.

\section{REVISÃO BIBLIOGRÁFICA}

\section{A dor na sala de recuperação pós-anestésica}

A SRPA é o local destinado a receber pacientes em pós-operatório imediato submetidos às anestesias geral e/ou regional, onde são implementados cuidados intensivos até o momento em que o paciente esteja consciente, com reflexos protetores presentes e com estabilidade dos sinais vitais. É o local onde o paciente 
permanece após o procedimento cirúrgico, sob a observação e cuidados constantes da equipe de enfermagem, como avaliação dos sinais vitais, nível de consciência, cuidados com ferida operatória, avaliação da dor, realizando ações preventivas a complicações decorrentes do ato cirúrgico (BONETTI AEB, et al., 2017).

As principais complicações que podem ocorrer na SRPA são náuseas e vômitos, alteração do nível de consciência, hipoxemia, hipotensão, hipertensão e arritmias cardíacas. Além de distúrbios emocionais como: ansiedade, medo, irritabilidade, raiva e depressão (BARBOSA MH, et al., 2014).

A dor é definida como uma experiência sensorial e emocional desagradável associada à lesão dos tecidos, podendo ser aguda ou crônica, causar sequelas e levar ao risco de morte (KAWAI VFA, et al., 2015; SILVA JA e RIBEIRO NP, 2011; CORREIA MDL e DURAN ECM, 2017). Relatada como sendo a pior experiência da vida, podendo ter fatores associados de complicações físicas e emocionais, podendo agravar a doença que motivou a cirurgia (PAULA GR, et al., 2011).

A dor no pós-operatório é subjetiva e pessoal, um evento natural de proteção do corpo quando qualquer tecido for lesionado, fazendo com que o indivíduo reaja para remover o estímulo doloroso. A incidência, intensidade e localização dependem de fatores individuais, como ansiedade, medo, depressão, fatores genéticos e ambientais, o tipo de cirurgia, a extensão do trauma, a gravidade do procedimento, doenças préexistentes e o método analgésico prescrito (SOARES AB, et al., 2013).

A dor pode estar relacionada à frustração, raiva e depressão, crença, expectativa e percepção do paciente na sua condição. A dor no pré-operatório, a duração da doença e a ansiedade pós-cirúrgica são considerados preditivos para o desenvolvimento de dor crônica no pós-operatório de cirurgia ortopédica (PEREIRA DL, et al., 2016). A dor no pós-operatório pode causar danos e sofrimento ao corpo, acometer os sistemas pulmonar, cardiovascular, gastrointestinal, endócrino e imunológico, prejudicando os pacientes que já apresentem algum tipo de agravo à saúde em decorrência da idade, doença ou lesão. (SOARES AB, et al., 2013).

O estímulo doloroso prolongado pode retardar a melhora do paciente, gera cansaço, insônia, fadiga, estresse, prejuízo da movimentação e deambulação, alteração de humor e desesperança da vida. Resultados mostram que o controle da dor é importante para reduzir as alterações fisiológicas, melhorar a qualidade de vida e aperfeiçoar a assistência prestada ao paciente (BARBOSA MH, et al.,2014). Pacientes que são submetidos a cirurgias abdominais, ortopédicas ou torácicas podem apresentar maiores índices de dor no pós operatório, já os pacientes que sofreram cirurgias de longa duração, relacionadas ao câncer ou de emergência podem requisitam mais analgesia nos períodos pós cirurgia (MORO ET, et al., 2016).

\section{Pós-operatório em cirurgias ortopédicas}

Nesse período são coletados dados do paciente referentes ao procedimento cirúrgico, tipo de anestesia, analgesia, curativos, drenos e posicionamento. A equipe de enfermagem observa o nível de consciência, o despertar, localização no tempo e espaço e se responde aos estímulos táteis e verbais. Na cirurgia ortopédica há incidência de delirium pós-operatório, principalmente em idosos (BARBOSA MH, et al., 2014).

Os procedimentos cirúrgicos têm finalidade de melhorar a funcionalidade, restaurando o movimento e a estabilidade, proporcionando o alívio da dor. As alterações musculoesqueléticas mais frequentes são: fraturas não estabilizadas, deformidades, doenças articulares, tecidos necrosados ou infectados, traumas e tumores (SOARES AB, et al., 2013).

A dor é um dos principais fatores que limitam a deambulação e aumenta o risco de trombose venosa profunda (TVP) pela imobilidade; além de alterações metabólicas que afetam outros sistemas. Sendo assim, é fundamental o manejo individualizado com uso de técnicas analgésicas adequadas que auxiliam na recuperação pós-operatória, reduz o tempo de internação e acelera o retorno às atividades de vida diária (DIAS AS, et al., 2016; PAULA GR, et al., 2011).

Devido à imobilidade física, a integridade cutânea também deve ser avaliada para prevenir lesão por pressão. O risco de TVP é alto em cirurgias ortopédicas de membros inferiores e ocorre entre o $5^{\circ}$ e $7^{\circ}$ dia do pós-operatório devido ao comprometimento e mobilidade prejudicada (BARBOSA MH, et al., 2014). 
É importante tratar a dor aguda pós-operatória para que não corra risco de desenvolver dor crônica e aumento da morbidade. A dor reduz a deambulação precoce e favorece o aparecimento de TVP, principalmente em pacientes idosos e submetidos à cirurgia de grande porte (BIDESE BL, et al., 2014).

A infecção é uma grave complicação particularmente em pacientes idosos, obesos, desnutridos, portadores de diabetes, artrite reumatóide, infecções concomitantes ou grandes hematomas (SOARES AB, et al., 2013).

Os pacientes ortopédicos sentem dor devido à lesão tecidual gerada no trauma, avaliação inadequada da dor e uso inadequado de analgésicos. Isso dificulta o tratamento e a recuperação, havendo alterações nos sinais vitais (KAWAI VFA, et al., 2015). Os pacientes de pós-operatório de cirurgias ortopédicas apresentam maior dor do que outros pacientes de outras especialidades. A característica dessa dor pode variar, sendo referida pela maioria dos pacientes como moderada a forte nas primeiras 24 horas do pós-operatório. Mulheres têm menor limiar de dor e resposta diferente ao estímulo álgico que os homens (BIDESE BL, et al., 2014).

Em cirurgias de membros inferiores a anestesia neuroaxial tem mais benefícios no pós-operatório, comparada à anestesia geral. Ela alivia a dor, permite mobilização precoce e diminui infecções, complicações pulmonares e internação hospitalar (PEREIRA DL, et al., 2016).

O controle da dor no período pós-operatório compreende diversas técnicas, desde métodos mais simples como prescrição de medicamentos analgésicos, até os mais sofisticados como a utilização de cateteres peridurais. Todos esses métodos têm em comum a intenção de evitar o sofrimento do paciente, aumentando a sua satisfação com o atendimento prestado, reduzindo o risco de complicações que resultam em aumento de custos resultantes de um período prolongado de internação (BARBOSA MH, et al., 2014; ROBLEDA G, et al., 2014).

\section{Analgesia}

Na SRPA os sinais vitais são muito importantes para direcionar a sensibilidade álgica, pois a dor repercute em valores da pressão arterial, frequências cardíaca e respiratória e na temperatura corporal. Alterações como sudorese, palidez cutânea, náuseas e vômito também são indicativos de dor (BARBOSA MH, et al., 2014). A analgesia é muito importante para a recuperação do paciente, pois a dor pode levar a inúmeras alterações na fisiologia do organismo, também afeta a deambulação e a movimentação precoce, dificulta 0 sono, o que leva a desgaste físico, fadiga e falta de adesão ao tratamento (BARBOSA MH, et al., 2014).

Segundo a Sociedade Brasileira para o Estudo da Dor (2013) o atendimento ao paciente com dor é de fundamental importância, pois interfere nos outros sinais vitais. A dor é considerado como o quinto sinal vital, juntamente com a frequência cardíaca, frequência respiratória, pressão arterial e temperatura. $\mathrm{O}$ controle da dor melhora a atividade cardiorrespiratória, evita o tromboembolismo, diminui a ansiedade e depressão e traz conforto para o paciente (RONDINELL MC, et al., 2016; SALGADO PO, et al., 2017; VIANA DL, et al., 2006).

O primeiro passo no controle da analgesia é a mensuração da dor, que merece a atenção de toda equipe de saúde. A cirurgia ortopédica é a principal causa de dores intensas em procedimentos de caráter ambulatorial. Observa-se que a maior incidência de pacientes submetidos a cirurgias ortopédicas, são do sexo masculino, com idade entre 18 a 93 anos, tendo a localização do procedimento nos membros inferiores (BARBOSA MH, et al., 2014).

Existem diferente métodos para avaliar a dor, em alguns instrumentos a dor é avaliada de forma qualitativa simples, variando apenas na intensidade. Já em outros instrumentos, a dor tem características multidimensionais, sendo considerada uma experiência composta também por fatores afetivos, emocionais e sensitivos (NASCIMENTO LA e KRELING MCGD, 2011).

A dor aguda pós-operatória é decorrente do trauma cirúrgico local que provoca alterações fisiológicas e psicológicas negativas, como a ansiedade e depressão que podem estar presentes desde o pré-operatório. O uso crônico de opióides faz com que se desenvolva tolerância ao fármaco e interferindo no controle adequado da dor, além da falta de treinamento, avaliação incompleta e desconhecimento dos possíveis efeitos colaterais dos analgésicos, pela equipe assistencial do paciente (BIDESE BL, et al., 2014). 
O uso de analgesia controlada pelo paciente (PCA) é considerado seguro e eficaz no tratamento da dor intensa a moderada e é frequentemente usado por pacientes com dor aguda no pós-operatório de cirurgia ortopédica (DIAS AS, et al., 2016) , a analgesia controlada pelo paciente e a analgesia epidural são prescritas para aliviar a dor, além de elevação do membro operado e aplicação de compressas frias para diminuir o edema (SMELTZER SC e BARE BG, 2012).

Podem ocorrer efeitos adversos como: hipotensão, retenção urinária e bloqueio motor, e em grandes doses de opioides resulta em sedação, depressão respiratória, constipação, confusão, náusea, vômito e prurido. A depressão respiratória é a mais preocupante, pois a infusão é por meio contínuo de bombas de infusão e o paciente pode estar sonolento ou sedado e não perceber os sintomas (DIAS AS, et al., 2016).

Para Kawai VFA, et al. (2015); os pacientes com algum analgésico prescrito no pré e no pós-operatório resulta em um fator positivo, pois ajuda a diminuir o estresse sofrido na internação, reduz o índice de infecção, os custos e re-internações, além de promover a reabilitação precoce.

As principais drogas prescritas são anti-inflamatórios não esteroides (AINE), analgésicos simples e opióides. O uso concomitante de AINE com outros medicamentos reduz a dose de opióides para o adequado controle da dor, sendo benéfico ao paciente que faz uso de vários fármacos (BARBOSA MH, et al., 2014).

A Organização Mundial de Saúde (OMS) recomenda uma prescrição que se adapte a cada necessidade do paciente, intensidade da dor, contra indicações e características de cada droga, sendo mais utilizadas a combinação de AINE, analgésicos simples e opioides administrados em horário fixo para o controle da dor e redução da ansiedade no pós-operatório (BARBOSA MH, et al., 2014).

A morfina é o opióide mais prescrito no tratamento de dor moderada a intensa e seu uso nas primeiras 24 horas do pós-operatório ortopédico está associado a menores taxas de queixas de dor (KAWAI VFA, et al., 2015). Estudos identificam dois fatores preditivos de dor imediata no pós-operatório: idade e ansiedade préoperatória. Por isso, pacientes jovens e/ou ansiosos devem receber analgesia diferenciada e que proporcione maior alívio da dor e da ansiedade (GEMMA R, et al., 2014; ROBLEDA G, et al., 2014).

O enfermeiro é o principal responsável pelo cuidado, promoção de conforto e alívio da dor no pósoperatório, pois tem um maior contato com o paciente e passa confiança à equipe médica. Ele colabora de forma positiva e coerente na adequação da analgesia, principalmente na modalidade da prescrição médica "se necessário", pois a avaliação e administração de fármacos exige conhecimento, responsabilidade profissional e ética (KAWAI VFA, et al., 2015).

Uma avaliação pré-operatória adequada melhora a assistência ao paciente e manejo da dor, propiciando um atendimento capacitado da equipe e recursos existentes, torna-se essencial para melhorar a qualidade de vida e manter o bem estar reduzindo também o tempo e custos da internação (BARBOSA MH, et al., 2014).

A prevenção, detecção e tratamento em tempo hábil auxiliam a reduzir os problemas de saúde associados à alta prevalência e intensidade de dor pós-operatória. Medo e ansiedade são dois fatores emocionais que, se reconhecidos corretamente, auxiliam na analgesia mais apropriada para cada paciente. A ausência de dor no pós-operatório é um indicador importante na satisfação do paciente com relação à cirurgia (ROBLEDA G, et al., 2014).

A dor pode ser intensa no pós-operatório de cirurgia ortopédica. Edema, hematomas e espasmos musculares podem contribuir para dor. A enfermeira avalia a dor, a resposta do paciente e as medidas terapêuticas e auxilia em esforços para aliviar a dor e os desconfortos (SMELTZER SC e BARE BG 2012).

Se for realizada de maneira efetiva, a analgesia auxilia na recuperação, aumenta a adesão do paciente ao tratamento levando a melhores resultados no pós-operatório. O enfermeiro possibilita que o paciente exponha suas preocupações e sentimentos do processo cirúrgico como um todo (BARBOSA MH, et al., 2014).

Para Barbosa MH, et al. (2014); o enfermeiro e a equipe de enfermagem são qualificados em identificar e prestar a melhor assistência ao paciente no pós-operatório, pois a dor é individual, subjetiva e complexa, um desafio aos profissionais de saúde em mensurá-la. 
O estado emocional do paciente é avaliado no momento da entrevista pré-operatória, onde ansiedade, sudorese, estresse, medo e choro podem estar presentes. Autores relatam que pacientes submetidos a cirurgias de grande porte como artroplastia de quadril, joelho ou ombro, referiram dor de maior intensidade na SRPA e nas primeiras 24 horas do pós-operatório, do que em outras cirurgias traumatológicas; e submetidos à anestesia geral sentiram mais dor do que a anestesia combinada ou local (ROBLEDA G, et al., 2014).

Ainda segundo Robleda G, et al. (2014); existe uma associação entre a ansiedade pré-operatória e a dor pós-operatória, onde muitos autores concordam a respeito da necessidade de melhorar a terapia da dor. Destacaram ansiedade, idade e tipo de cirurgia como os mais importantes fatores preditivos de dor pósoperatória e consumo de analgésicos, porém, não ficou claro se a ansiedade causa a dor ou se a dor causa a ansiedade.

O movimento e posicionamento do membro operado devem ser realizados de modo seguro e coordenado, observando presença de sinais flogísticos ou sangramento no curativo e local de drenos, pois a dor pode ser também sinal de infecção (SOARES AB, et al., 2013).

Disfunções orgânicas como hipoventilação, aumento do trabalho cardíaco, diminuição da perfusão sanguínea periférica e contração muscular reflexa estão associadas à persistência da dor; a incidência de náuseas e vômito, o tipo de anestesia realizada e os efeitos adversos também podem estar relacionados. $O$ tratamento adequado da dor aguda no pós-operatório reduz o sofrimento do paciente e diminui as morbidades associadas (BIDESE BL, et al., 2014).

Conforme Bidese BL, et al. (2014); a adequada analgesia pós-operatória pode ter início no pré-operatório com métodos a ser escolhidos de acordo com a intensidade de dor e conhecimento dos benefícios e efeitos adversos, por parte da equipe assistente. Esses métodos inibem as vias nociceptivas por meio da intervenção farmacológica como analgésicos orais e injeções intramusculares, analgésicos de ação periférica e central como os AINE e opióides e anestésicos para uso em bloqueios como PCA, analgesia peridural controlada ou não pelo paciente e bloqueio regional do nervo.

\section{Enfermeiro no cuidado da dor}

Faz parte das atividades da equipe de enfermagem a avaliação e registro da dor informando o local, a intensidade, a característica, a duração, e fatores que pioram ou melhoram, além da administração ou não de analgésicos (NASCIMENTO LA e KRELING MCGD, 2011).

Cabe ao enfermeiro estabelecer os diagnósticos e prescrever as intervenções de enfermagem que condizem com o estado do paciente e que auxiliam no alívio da dor, minimizando os seus efeitos e contribuindo para o cuidado do humanizado aos pacientes (SALGADO PO, et al., 2017).

A International Association for the Study of Pain lançou no ano de 2017 uma iniciativa global contra a dor pós-cirúrgica. Com intuito de promover a conscientização e incentivar melhores práticas no tratamento da dor no pós-operatório. Dentre essas práticas comunicação realizada durante a passagem de plantão pela enfermagem possibilita a observação da evolução da dor, auxiliando na analgesia. Esse processo também garante a segurança do paciente levando a melhores resultados pós cirúrgicos (BONETTI AEB, et al., 2017).

$\mathrm{Na}$ avaliação do paciente na sala de recuperação, verifica-se se os sinais vitais estão regulares, observando também o nível de consciência e os seus reflexos, avaliando seu retorno aos parâmetros normais, se ocorrência de náuseas e vômitos, orientação no tempo e espaço, a saturação de oxigênio, se existência de algum sangramento ativo na ferida operatória, observar se dor está controlada (CASTRO FSF, et. al., 2012).

No tratamento da dor, esse deve ser fundamentado em razões subjetivas, objetivando amenizar as respostas físicas e psicológicas causados pela cirurgia (POPOV DC e PENICHE AC, 2009). Diferentes pesquisas ainda apontam que a prevenção da dor pós-operatória é extremamente importante objetivando maximizar o resultado anestésico-cirúrgico, assim como objetivando a redução do sofrimento e o trauma relacionados ao tempo de internação hospitalar (MATTIA AL, et. al., 2013; CAPELLO RG, et. al., 2009). 


\section{CONSIDERAÇÕES FINAIS}

Identificou-se que a avaliação pré-operatória adequada melhora a assistência ao paciente e auxilia no manejo da dor, propiciando um atendimento capacitado da equipe e dos recursos existentes, torna-se essencial para melhorar a qualidade de vida e manter o bem-estar do paciente, reduzindo também o tempo e custos da internação. A avaliação da dor, analgesia efetiva, prevenção de complicações, ensino do autocuidado fazem parte do cuidado pós-operatório que levam a uma recuperação mais tranquila para 0 paciente. $O$ enfermeiro possibilita que o paciente exponha suas preocupações e sentimentos do processo cirúrgico como um todo, possuindo papel fundamental para avaliar, controlar e aliviar a dor no tratamento eficaz do trauma, pois acelera o processo de reabilitação e contribui na manutenção das funções fisiológicas do paciente.

\section{REFERÊNCIAS}

1. BARBOSA MH, et al. Avaliação da intensidade da dor e analgesia em pacientes no período pós-operatório de cirurgias ortopédicas. Esc. Anna Nery, 2014; 18 (1): 143-147.

2. BARROS EA. Ajustar-se, criativamente, é preciso: experiências e enfrentamentos em leitos da pré-cirurgia ortopédica. Rev NUFEN, 2018; 10(2).

3. BIDESE BL, et al. Analgesia pós-operatória por não especialistas em dor. Rev. dor, 2014; 15(1): 36-40.

4. BONETTI AEB, et al. Assistência da equipe de enfermagem ao paciente em sala de recuperação pós-anestésica. Rev Enferm UFSM. 2017; 7(2):193-205.

5. CAPELLO RG, et al. Intervenções de enfermagem na recuperação anestésica: controle da dor, náuseas, hipotermia e outras complicações do pós-operatório. Rev Dor. 2009.

6. CASTRO FSF, et al. Temperatura corporal, índice Aldrete e Kroulik e alta do paciente da Unidade de Recuperação Pós-Anestésica. Rev. Esc. Enferm. USP, 2012; 46(4): 872-876.

7. CORREIA MDL, DURAN ECM. Conceptual and operational definitions of the components of the nursing diagnosis Acute Pain. Rev. Latino-Am. Enfermagem, 2017; 25: e2973.

8. DIAS AS, et al. The impact of patients controlled analgesia undergoing orthopedic surgery. Brazilian Journal of Anesthesiology (English Edition). 2016; 66(3): 265-271.

9. GEMMA R, et al. Influence of preoperative emotional state on postoperative pain following orthopedic and trauma surgery. Rev. Latino-Am. Enfermagem, 2014; 22(5): 785-791.

10. KAWAI VFA, et al. Analgesia dos períodos pré e pós-operatório em cirurgias ortopédicas. Rev. Dor, 2015 ; $16(3)$ : 166 170.

11. MATTIA AL, et al. Infusão venosa aquecida no controle da hipotermia no período intraoperatório. Rev Latino-Am Enfermagem. 2013

12. MORO ET, et al. Qualidade da recuperação da anestesia em pacientes submetidos à cirurgia ortopédica em membros inferiores. Brazilian Journal of Anesthesiology (English Edition), 2016; 66 (6): 642-650

13. NASCIMENTO LA, KRELING MCGD. Avaliação da dor como quinto sinal vital: opinião de profissionais de enfermagem. Acta paul. enferm. 2011; 24(1): 50-54.

14. PAULA GR, et al. Assistência de enfermagem e dor em pacientes ortopédicos na recuperação anestésica, no Brasil. Revista Dor. 2011; 12(3): 265-269.

15. PEREIRA DL, et al. Dor após cirurgia eletiva ortopédica de grande porte em membro inferior e o tipo de anestesia: isso importa?. Rev. Bras. Anestesiol, 2016; 66(6): 628-636.

16. POPOV DC, PENICHE AC. As intervenções do enfermeiro e as complicações em sala de recuperação pós-anestésica. Rev Esc Enferm USP. 2009; 43(4): 953-61

17. ROBLEDA G, et al. Influência do estado emocional pré-operatório na dor pós-operatória após cirurgias ortopédicas e traumatológicas. Rev Latino-Am Enfermagem, set/out 2014, 22(5):785-791.

18. RONDINELLI MC, et al. Implementação de um programa de controle da dor em hospital de traumatologia e ortopedia. Relato de caso. Rev. dor. 2016, São Paulo; 17(2): 141-144.

19. SALGADO PO, et al. Características definidoras do diagnóstico de enfermagem "dor aguda" em pacientes no pósoperatório imediato. Enf revista.2017.20(3):66-79.

20. SILVA JA, RIBEIRO NP. A dor como um problema psicofísico. Rev. dor, São Paulo.2011;12(2):138-151.

21. SOARES $A B$, et al. A assistência de enfermagem ao paciente submetido à artroplastia total de quadril e a importância dos cuidados no pós-operatório. Revista Científica de Enfermagem, São Paulo. 2013;3 (7):11-18.

22. SOCIEDADE BRASILEIRA PARA O ESTUDO DA DOR. Hospital sem dor diretrizes para implantação da dor como 5은 sinal vital. São Paulo, 2013.

23. SMELTZER SC, BARE BG. Tratado de Enfermagem médico cirúrgica. 12.ed. Rio de Janeiro: Guanabara Koogan, 2012:2050.

24. VIANA DL, et al. A avaliação da dor da criança pelas enfermeiras na Unidade de Terapia Intensiva. Pediatria (São Paulo). São Paulo. 2006, 28(4): 251-261. 